\title{
Caracterización de semielaborados de aleaciones de titanio procesadas por extrusión y laminación perforación (proceso Mannesmann)
}

\section{Characterization of semi-finished titanium alloys processed by extrusion and lamination perforation (Mannesmann process)}

\author{
${ }^{1}$ Fabricación de Aleaciones Especiales S. A. (FAE S. A.), Pbto. J. González y Aragón 15,(1802) Ezeiza, Buenos Aires, \\ Argentina \\ e-mail: claudio_bunte@yahoo.com \\ ${ }^{2}$ Universidad Tecnológica Nacional, Facultad Regional Buenos Aires, Dto Ing. Industrial, Medrano 951, (1179) CABA, \\ Buenos Aires, Argentina \\ ${ }^{3}$ Universidad Nacional de Gral San Martín, Inst. de Tecnología “Jorge Sábato”, Av. Gral. Paz 1499, (1650) San Martín, \\ Buenos Aires, Argentina
}

\section{RESUMEN}

Para la fabricación de tubos y caños sin costura se utilizan como materias primas, semielaborados huecos provenientes de procesos de deformación en caliente tales como extrusión y laminación perforación (proceso Mannesmann). A partir de los cuales y por medio de sucesivas reducciones de área a través de laminación o de trafilación se alcanzan las medidas finales solicitadas. El proceso de laminación perforación, por efecto Mannesmann, contempla la perforación de tochos cilíndricos, a través de una compresión diametral progresiva, que genera una fractura en el núcleo y facilita la perforación junto a la laminación transversal oblicua. En cambio la extrusión es un proceso por compresión en el cual el metal es forzado a fluir a través de una matriz para darle forma a su sección transversal. El presente trabajo de caracterización se realizó sobre semielaborados provenientes de ambos procesos, laminación perforación y extrusión, en aleaciones de titanio (Titanio Gr. 2 (Titanio Comercialmente Puro) y Titanio Gr. 5 (Ti-6Al-4V)). Se realizaron ensayos destructivos (tracción, aplastado y abocardado), no destructivos (inspección visual, videoscopía, dimensionales, rugosidad superficial y determinación de discontinuidades por tintas penetrantes) y macro y micro metalografías. También se evaluó la contaminación química provocada por la atmósfera del horno. Finalmente se analizaron los defectos característicos provenientes de cada proceso de deformación, su causa raíz y sus posibles soluciones.

Palabras clave: Deformación en caliente, Laminación perforación, Extrusión, Aleaciones de titanio.

\section{ABSTRACT}

For the manufacture of seamless pipes and tubes are used, as raw materials, hollow bars from hot deformation processes like extrusion and rotary tube piercing (Mannesmann process). Starting from these semielaborated products and by means of successive cross sections reductions through tube drawing or tube rolling (cold pilgering) the requested final dimensions are achieved. The rotary tube piercing process, Mannesmann process, includes drilling cylindrical billet through a progressive diametral compression that generates a crack at the center and facilitates perforation along the oblique transverse rolling. In contrast, the extrusion is a compression process in which the metal is forced to flow through a die to shape cross section. This characterization work was performed on semifinished products from both processes, extrusion and rotary tube piercing in titanium alloys (Titanium Gr. 2 (Titanium Commercially Pure) and Titanium Gr. 5 (Ti-6Al-4V)). Destructive tests (tensile test, flattening test and flaring test) non-destructive tests (visual inspection, boroscopy, dimensional, surface roughness and discontinuity determination by dye penetrant) and metallography (macro and micro) were performed. Chemical contamination caused by the furnace atmosphere was also assessed. Finally, the characteristic defects from each deformation process, their root cause and possible solu- 
tions were discussed.

Keywords: Hot deformation, Rotary tube piercing, Extrusion, Titanium alloys.

\section{INTRODUCCIÓN}

\subsection{Trabajabilidad en caliente}

La trabajabilidad en caliente de un metal o aleación es la capacidad para ser deformado bajo condiciones de alta temperatura y relativamente elevadas velocidades de deformación sin que se produzcan fallas ni roturas. Las dos características que gobiernan a la trabajabilidad en caliente son la resistencia a la deformación y la ductilidad. A su vez éstas dependen de las variables de proceso y de las variables metalúrgicas. Dentro de las variables del material que afectan a la trabajabilidad tenemos a los mecanismos de fractura, al flujo localizado y a consideraciones metalúrgicas [1].

Durante el conformado plástico de lingotes o semielaborados de colada continua y los subsiguientes pasos de deformación, la segregación, las partículas de segunda fase, las inclusiones y los mismos granos de la estructura cristalina se alinean en la dirección de mayor flujo de material (líneas de flujo), produciendo variaciones direccionales en las propiedades tales como resistencia, ductilidad, resistencia al impacto y fatiga $[1,3]$.

Los parámetros dependientes del proceso son la deformación, la velocidad de deformación, la temperatura, la fricción, el estado tensional y los criterios de fluencia [2].

La trabajabilidad de un material puede ser representada a través de los mapas de trabajabilidad, que son gráficas que representan la zona segura para la deformación en función de la velocidad de deformación real y la temperatura del material y las zonas de ocurrencia de fallas [3]

\subsection{Titanio y Sus Aleaciones}

El titanio puro posee una transformación alotrópica de una fase $\alpha$ (hcp) de baja temperatura a una fase $\beta$ (bcc) a una temperatura de $882^{\circ} \mathrm{C}$. Al agregar aleantes esta temperatura de transformación puede aumentar o descender, según los elementos agregados sean alfágenos $(\mathrm{O}, \mathrm{N}, \mathrm{C}, \mathrm{Al}, \mathrm{Si})$ o betágenos $(\mathrm{V}, \mathrm{Mn}, \mathrm{Mo}, \mathrm{Nb}, \mathrm{Zr}$, $\mathrm{H}, \mathrm{Fe}$ ), es decir que estabilizan una fase u otra, de esta forma pueden caracterizarse las aleaciones de titanio en tres familias, las aleaciones $\alpha$, las aleaciones $\beta$ y las aleaciones $\alpha+\beta[\underline{4}, \underline{6}]$.

El titanio puro y las aleaciones $\alpha$, presentan estructura cristalina (hcp) a temperaturas ambiente. Estas aleaciones poseen buena resistencia a la tracción y al creep, buena soldabilidad y ductilidad.

Las aleaciones $\beta$, en su mayoría son aleaciones de titanio con otros metales de transición, ya que éstos estabilizan la fase fcc, razón por la cual estas aleaciones son fácilmente conformables.

Finalmente las aleaciones $\alpha+\beta$ mantienen un equilibrio entre ambas fases. Un ejemplo de estas aleaciones es el Titanio Gr. 5 (Ti-6Al-4V). Generalmente presentan buena formabilidad y buena resistencia a temperatura ambiente, pasando a ser de moderada resistencia a altas temperaturas. A diferencia de las anteriores en este tipo de aleaciones las propiedades y las microestructuras pueden controlarse mediante tratamientos térmicos $[\underline{5}, \underline{6}]$.

\subsection{Procesos de Deformación}

Todos los procesos de deformación de los metales pueden clasificarse según los esfuerzos aplicados: compresión directa, compresión indirecta, tracción, doblado y corte. El método de deformación plástica, acompañado por correctos tratamientos térmicos, da lugar a procesos termomecánicos con el fin de obtener propiedades mecánicas específicas [2].

En este trabajo se hará una caracterización de productos provenientes de dos procesos, uno de compresión directa (laminación perforación mediante el efecto Mannesmann) y otro de compresión indirecta (extrusión). Los productos de ambos procesos son considerados como semielaborados para la fabricación de tubos sin costura.

\subsection{Laminación Perforación, Proceso Mannesmann}

El laminador perforador está formado por un par de cilindros laminadores bicónicos que giran en el mismo sentido y un mandril perforador. Los cilindros, cuyos ejes están alabeados en un ángulo variable $\phi\left(\right.$ de $8^{\circ}$ a $30^{\circ}$ ), están separados entre sí a una distancia mínima llamada garganta, dentro de la cual se posiciona el mandril de perforación. Dicho alabeo provoca una componente de velocidad tangencial de los cilindros que hace 
avanzar el tocho describiendo una trayectoria helicoidal. Debido al perfil de los cilindros a medida que el tocho avanza, éste va sufriendo una compresión diametral progresiva a medida que gira, lo cual provoca tensiones de tracción y compresión alternadas en el centro del material [ㄱ,9].

Los parámetros principales del proceso que afectan la calidad de los perforados, además de la temperatura y estructura del material, son: el ángulo entre cilindros, el perfil y la velocidad de giro de los mismos, la posición del mandril en la garganta y el lugar de contacto con el tocho [7].

\subsection{Extrusión}

La extrusión es un proceso de deformación utilizado para obtener productos largos, semielaborados o finales como tubos, perfiles o barras, donde un pistón presiona un billet, generalmente redondo contra una matriz de la forma deseada y a través de ella se obtiene el producto deseado. Según el movimiento relativo entre el pistón y el sentido de salida del extrudado se definen dos procesos de extrusión: extrusión directa e indirecta.

La materia prima de este proceso son billets, con o sin mecanizar y forjados o brutos de colada, que pueden estar lubricados o no. El calentamiento se realiza en hornos de inducción o de combustión.

Los parámetros principales del proceso que afectan la calidad de los extrudados además de los del material, son: la cantidad de deformación, el ángulo de la matriz, la velocidad de extrusión y la lubricación.

Los defectos más comunes causados por inestabilidades de flujo suelen ser la formación de embudo y de cañería o "pipe". Así mismo pueden presentarse otros defectos, como el de bambú, de excentricidad, falta de rectitud, mala textura superficial interior o exterior, entre otros.

Para evitar todos estos defectos existe gran variedad de métodos de extrusión, con y sin cáscara, con lubricación, con descarte de colada, etc. Cualquier modificación que disminuya el rozamiento entre el material y el herramental disminuirá la probabilidad de generar defectos $[\underline{10}, \underline{11}]$.

\section{DESARROLLO}

\subsection{Muestras de laminados perforados}

Los laminados perforados utilizados fueron obtenidos en el laminador perforador que posee el laboratorio del Centro de Investigaciones Industriales (CINI) del Grupo Techint, en la sede de Campana (Buenos Aires) de la empresa Tenaris. Este perforador de laboratorio simula dicho proceso y determina parámetros, extrapolables a escala industrial.

Los tochos de aleaciones de titanio utilizados tenían $35 \mathrm{~mm}$ de diámetro y $150 \mathrm{~mm}$ de largo y fueron fabricados por FAE S. A.. El Titanio Gr. 2 (o comercialmente puro) se procesó a una temperatura de $950^{\circ} \mathrm{C}$ mientras que el Titanio Gr. 5 a $1150^{\circ} \mathrm{C}$ y una vez perforados se dejaron enfriar al aire.

Los parámetros de operación del laminador fueron los mismos para las dos aleaciones (el ángulo de alimentación, la apertura de las zapatas guías, la velocidad de rotación de los cilindros, la velocidad de rotación y la posición de la lanza). La Figura 1 muestra los perforados obtenidos.

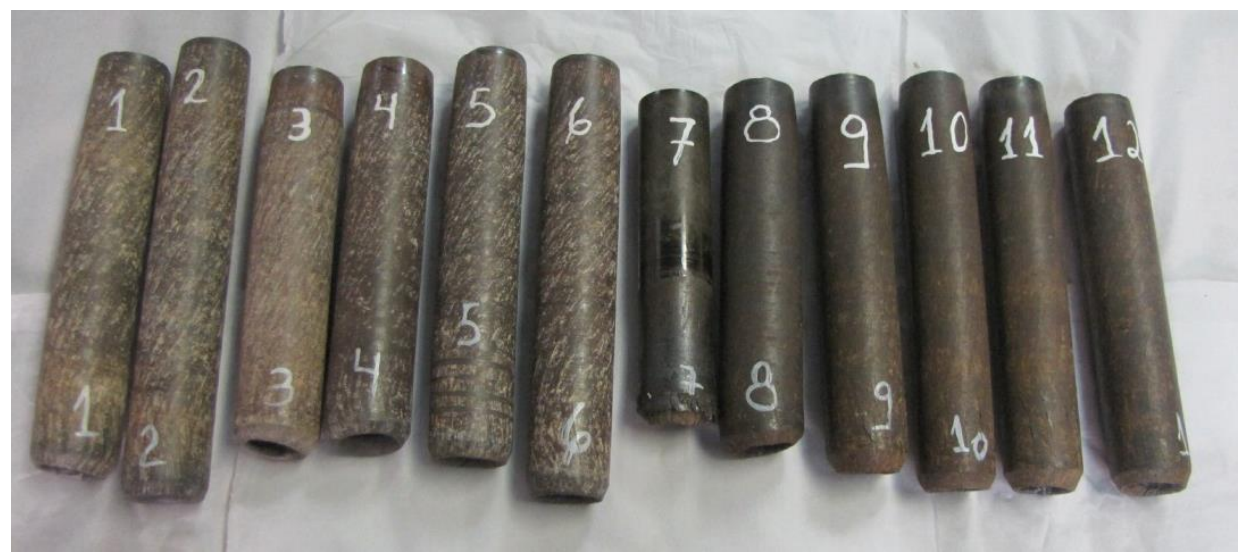

Figura 1: Semielaborados perforados. Izq.: Titanio Gr.2. Der.: Titanio Gr. 5.

\subsection{Muestras de extrudados}

FAE S. A. suministró tubos extrudados de Titanio Gr. 2, de dos medidas diferentes (Ø $91 \mathrm{~mm}$ x 12,5 mm de 
espesor у Ø $71 \mathrm{~mm}$ x 12,0 mm de espesor), provenientes de pruebas realizadas en distintos equipos.

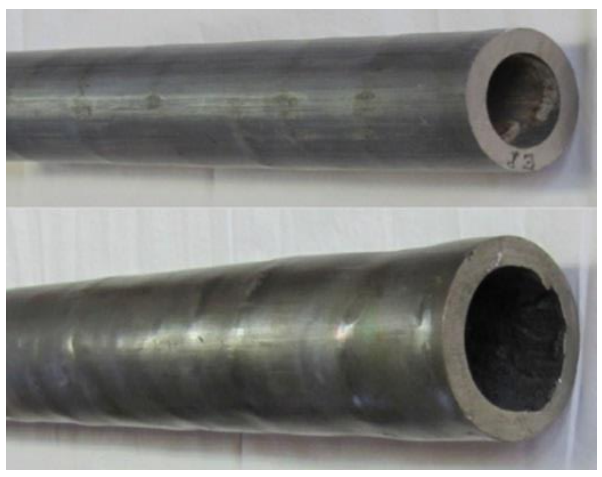

Figura 2: Semielaborados extrudados de Titanio Gr.2.

En ambos casos los billets, previa lubricación con solución de grafito coloidal, se calentaron en hornos de empuje a temperaturas entre $980{ }^{\circ} \mathrm{C}$ y $1020^{\circ} \mathrm{C}$. El tubo de mayor diámetro se extrudó en FV S. A.- Grifería de alta tecnología, con una prensa de $1000 \mathrm{t}$, una presión de trabajo de $190 \mathrm{~kg} / \mathrm{cm}^{2}$ y una velocidad de extrusión de $30 \mathrm{~mm} / \mathrm{s}$ aprox. Mientras que el segundo se procesó en Metalcris S. A., con una prensa de 700 t, una presión de trabajo de $390 \mathrm{~kg} / \mathrm{cm}^{2}$ y una velocidad de extrusión de $50 \mathrm{~mm} / \mathrm{s}$ aprox.. (Ver Figura 2).

\subsection{Caracterización}

Para la caracterización se utilizaron las técnicas y los ensayos siguientes:

a. Composición química

b. Inspección visual (ojo desnudo y videoscopio (Olympus IPLEX FX)),

c. Ensayo de tintas penetrantes (Tinta Spotcheck, SKL-WP y revelador Zyglo, ZP-9F, de Magnaflux)

d. Control dimensional (excentricidad y ovalidad)

e. Análisis metalográfico (macro y micro)

f. Ensayos de tracción (Instron modelo 1125 de 10 t.).

g. Medición de dureza (Durómetro Officine Galileo, modelo DG 302)

h. Ensayos de aplastado y abocardado (Prensa Schirmer+Plate, 2500 t.)

\section{RESULTADOS Y DISCUSIÓN}

\subsection{Composición química}

En la Tabla 1, se muestran las composiciones químicas de los semielaborados utilizados en este trabajo, informadas por FAE S. A. y que cumplen con las normas ASTM B-338 y ASTM B-861 [12, 13$]$.

Tabla 1: Composición química semielaborados.

\begin{tabular}{lcccccccc}
\hline \multicolumn{1}{c}{ Aleación } & $\begin{array}{c}\text { C } \\
\%\end{array}$ & $\begin{array}{l}\text { N } \\
\%\end{array}$ & $\begin{array}{l}\text { H } \\
\%\end{array}$ & $\begin{array}{l}\text { O } \\
\%\end{array}$ & $\begin{array}{c}\text { Fe } \\
\%\end{array}$ & $\begin{array}{l}\text { Al } \\
\%\end{array}$ & $\begin{array}{l}\text { V } \\
\%\end{array}$ & $\begin{array}{l}\text { Ti } \\
\%\end{array}$ \\
\hline $\begin{array}{l}\text { Titanio Gr. 2 } \\
\text { (laminado perforado) }\end{array}$ & 0.03 & 0.02 & 0.007 & 0.19 & 0.21 & -- & --- & Balance \\
\hline $\begin{array}{l}\text { Titanio Gr. 2 } \\
\text { (extrudado) }\end{array}$ & 0.02 & 0.02 & 0.009 & 0.20 & 0.25 & -- & --- & Balance \\
\hline $\begin{array}{l}\text { Titanio Gr. 5 } \\
\text { (laminado perforado) }\end{array}$ & 0.03 & 0.03 & 0.010 & 0.16 & 0.31 & 5.78 & 3.95 & Balance \\
\hline
\end{tabular}

En la Tabla 2 se muestran los valores del análisis químico de los gases en las capas superficiales (0,5 $\mathrm{mm}$ ), de ambos productos, para evaluar la contaminación gaseosa durante el calentamiento. 
Tabla 2: Composición química gaseosa, exterior, media e interior.

\begin{tabular}{lcccc}
\hline \multicolumn{1}{c}{ Aleación } & Elemento & $\begin{array}{c}\text { Diámetro } \\
\text { exterior } \\
\%\end{array}$ & $\begin{array}{c}\text { Diámetro } \\
\text { medio } \\
\%\end{array}$ & $\begin{array}{c}\text { Diámetro } \\
\text { interior } \\
\%\end{array}$ \\
\hline Titanio Gr. 2 & $\mathrm{H}$ & 0.0029 & 0.0010 & 0.0060 \\
(laminado perforado) & $\mathrm{O}$ & 0.3440 & 0.2020 & 0.2900 \\
\hline Titanio Gr. 2 & $\mathrm{N}$ & 0.0064 & 0.0019 & 0.0035 \\
(extrudado) & $\mathrm{H}$ & 0.0030 & 0.0029 & 0.0102 \\
\hline Titanio Gr. 5 & $\mathrm{O}$ & 0.2030 & 0.1890 & 0.2302 \\
(laminado perforado) & $\mathrm{N}$ & 0.0025 & 0.0020 & 0.0028 \\
\hline & $\mathrm{H}$ & 0.0056 & 0.0037 & 0.0067 \\
& $\mathrm{O}$ & 0.5780 & 0.1570 & 0.2510 \\
& $\mathrm{~N}$ & 0.0011 & 0.0052 & 0.0120 \\
\hline
\end{tabular}

Para la cáscara de extrusión el incremento de gases se manifestó sólo en el oxígeno cuyo resultado fue $1,42 \%$.

\subsubsection{Inspección visual}

En todos los laminados perforados se observó un rayado helicoidal (interior y exterior), característico del proceso (Figura 3). En algunos casos este rayado penetraba la capa de óxido superficial hasta $2 \mathrm{~mm}$.

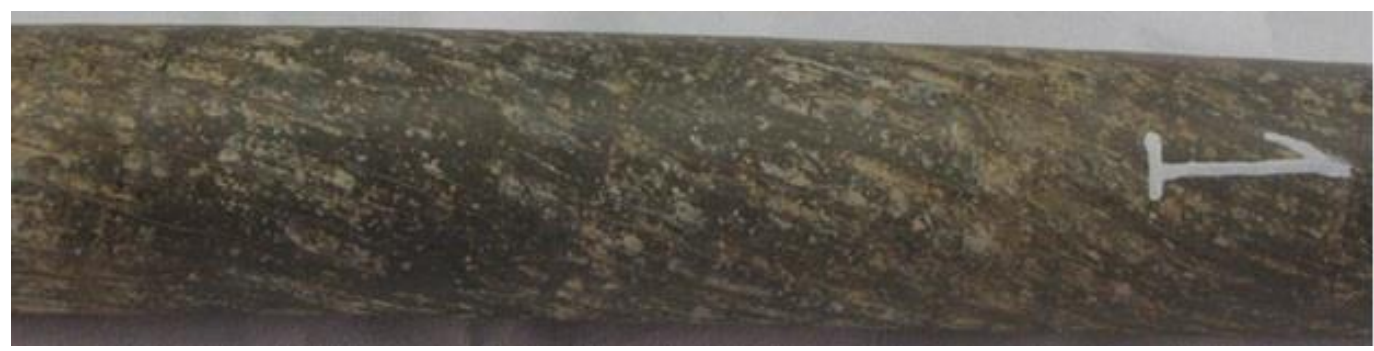

Figura 3: Rayado helicoidal, característico de la laminación helicoidal (proceso Mannesmann)

La videoscopía interior evidenció la deformación helicoidal y también la presencia de defectos como pliegues, rayas y fisuras (Figura 4).

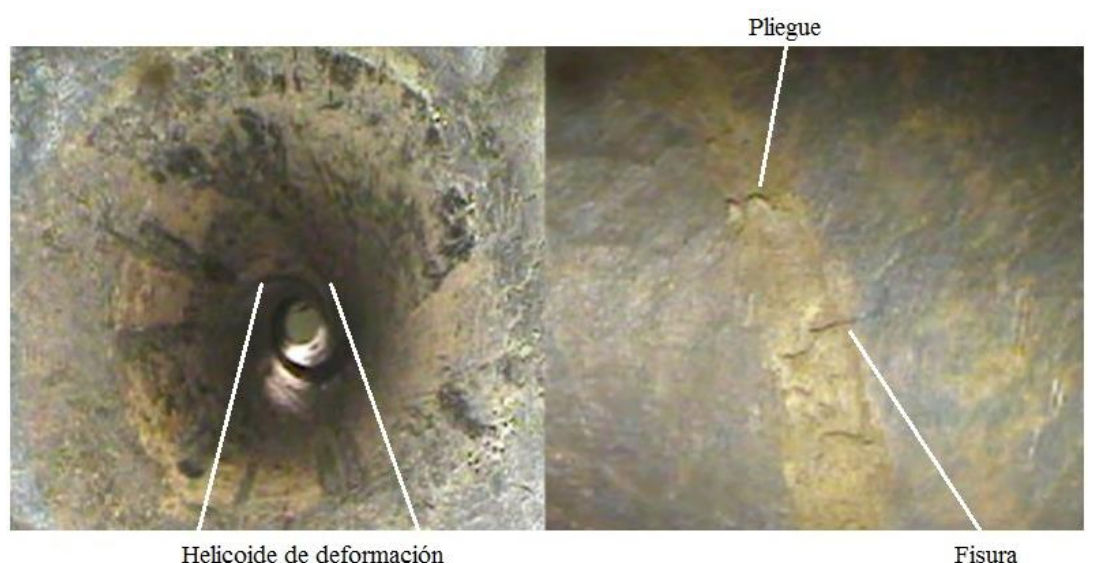

Figura 4: Defectos internos: Rayado helicoidal (Izq.) y Fisuras y pliegues (Der.).

Se aprecia una diferencia de coloración de la capa de óxido en las diferentes aleaciones. El Titanio Gr. 5 presenta un óxido más oscuro que el Titanio Gr. 2, debido a la presencia de los elementos aleantes.

En la inspección visual realizada a los extrudados, se encontraron varios defectos propios del proceso de extrusión, tales como rayas, escamas, "bambú”, material pegado, etc. (Figura 5). 

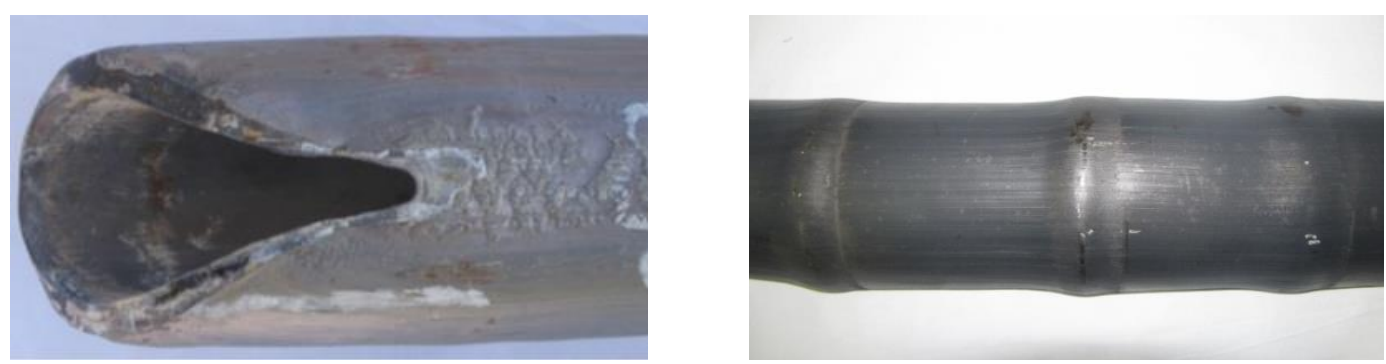

Figura 5: Quemado de material (Izq,). Defecto tipo "bambú" (Der.)

\subsubsection{Tintas penetrantes}

Las muestras de los laminados perforados analizadas, confirmaron que los pliegues encontrados en el Titanio Gr. 2 eran de menor magnitud que los de Titanio Gr. 5 como se muestra en la Figura 6. Notándose además en ambos casos una incidencia mayor al inicio de la perforación.

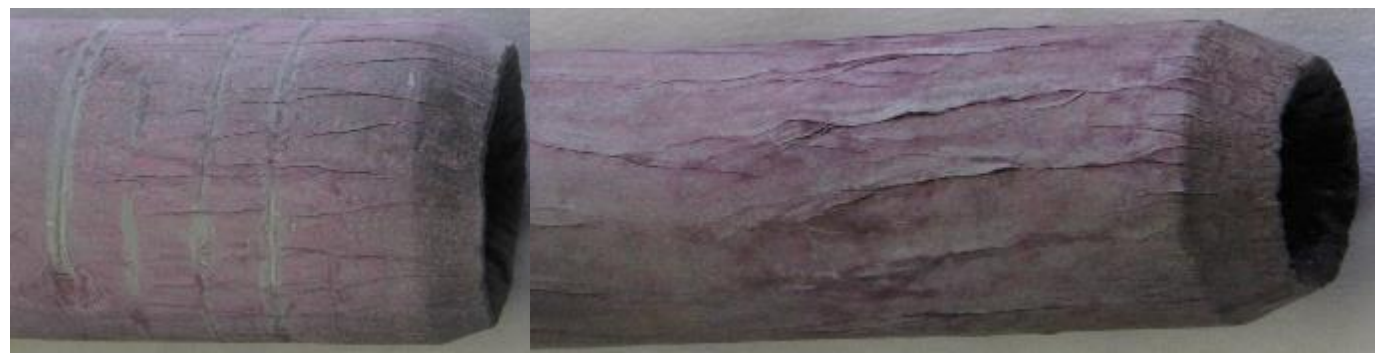

Figura 6: Tintas penetrantes en sección de entrada de dos perforados. Izq.: Titanio Gr.2. Der.: Titanio Gr. 5.

También se analizó la muestra de Titanio Gr. 5 que no perforada debido a la baja temperatura del material. No se encontraron indicios de la propagación de la fisura central (Efecto Mannesmann) (Figura 7).

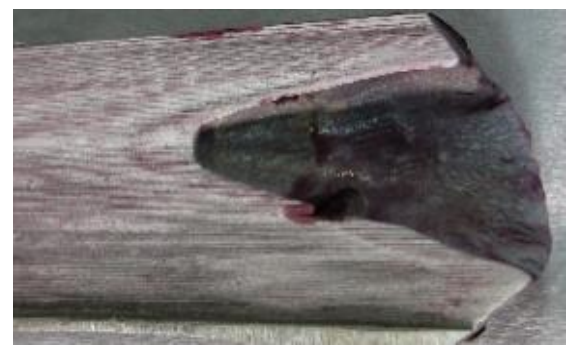

Figura 7: Tintas penetrantes en tocho parcialmente perforado.

En los extrudados, se aplicaron tintas penetrantes sobre dos piezas, una colada de extrusión y un corte longitudinal (extrudado de $91 \mathrm{~mm}$ de diámetro).

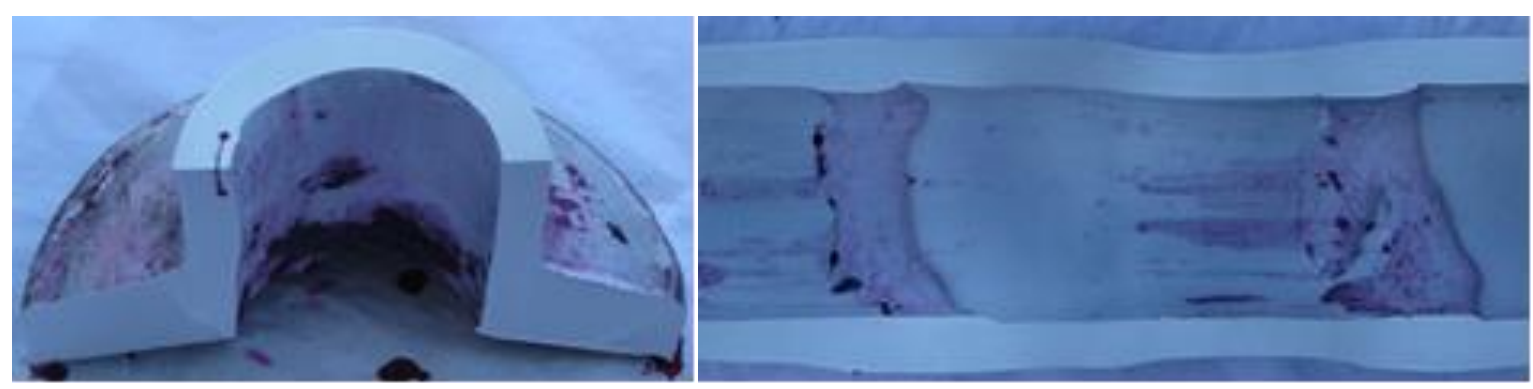

Figura 8: Tintas penetrantes. En colada de extrusión (Izq.). Defecto tipo “bambú” (Der.). 
En la Figura 8 (izq.) se encontró una fisura interna en la colada de extrusión y en el final del tubo extrudado. En la figura de la derecha, se detectaron fisuras en el interior del extrudado, en la zona de acumulación de material producida por el efecto "bambú".

\subsubsection{Control dimensional (excentricidad y ovalidad)}

En los laminados perforados, los valores de ovalidad, obtenidos para el Titanio Gr. 2 presentaron un máximo del $4 \%$ con un promedio del $2 \%$, mientras que los del Titanio Gr. 5 fueron $3 \%$ y $1.8 \%$ respectivamente. La excentricidad, si bien al comienzo de la perforación es alta, la misma se fue reduciendo conforme avanzaba la deformación. En el Titanio Gr. 2 la excentricidad inicial fue del 6\% y la final del 2\%. Para el Titanio Gr. 5 las muestras presentaron al inicio valores dispares entre el $40 \%$ y $20 \%$ pero terminando en el orden del $10 \%$.

En los extrudados, los valores de ovalidad obtenidos presentaron un máximo del $5 \%$ con un promedio del $1.8 \%$. En el caso de la excentricidad, las mediciones realizadas indicaron una excentricidad inicial entre $20 \%$ y el $30 \%$, reduciéndose a un $7.4 \%$ de promedio a partir de los $350 \mathrm{~mm}$ de la punta.

\subsubsection{Metalografías}

Las macrografías de los laminados perforados revelaron el flujo del material durante el proceso. También se verificó que en las macrografías transversales la microestructura del perforado de Titanio Gr. 2 es de menor tamaño que la de Titanio Gr. 5.

Las micrografías permitieron además de relevar las microestructuras presentes, caracterizar la capa de óxido superficial y medir la profundidad de varias fisuras transversales $[\underline{14}, \underline{15}]$.

Se notaron zonas afectadas por la deformación provocada por el herramental y un fibrado mecánico en dirección helicoidal. El tamaño de grano no pudo ser medido ya que no se encontraron granos equiaxiales.

La Figura 9 muestra un pliegue superficial y las microestructuras, exterior y central, de una sección longitudinal del tubo perforado.
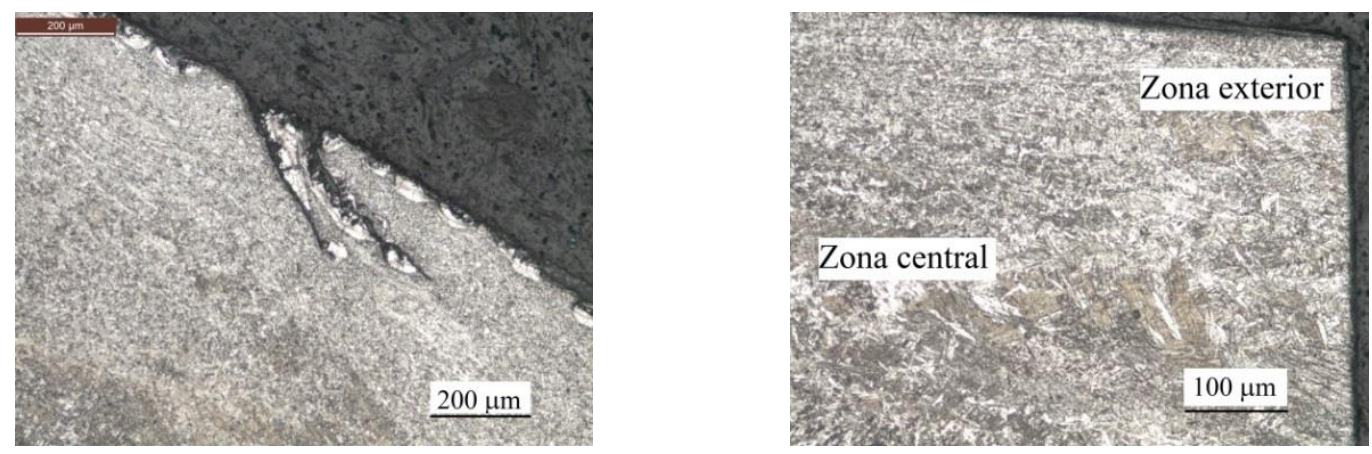

Figura 9: Pliegue de 0,25 mm de profundidad. 50X (Izq.) y Microestructuras de la zona exterior y central. 100X (Der.)

En la Figura 10, izquierda, no se aprecia claramente un fibrado mecánico debido a la extrusión. La microestructura resultante es la del procesamiento en fase $\beta$ con un enfriamiento rápido al aire, donde se obtuvo $\alpha^{\prime}$ (acicular). También puede apreciarse la capa superficial " $\alpha$ case".
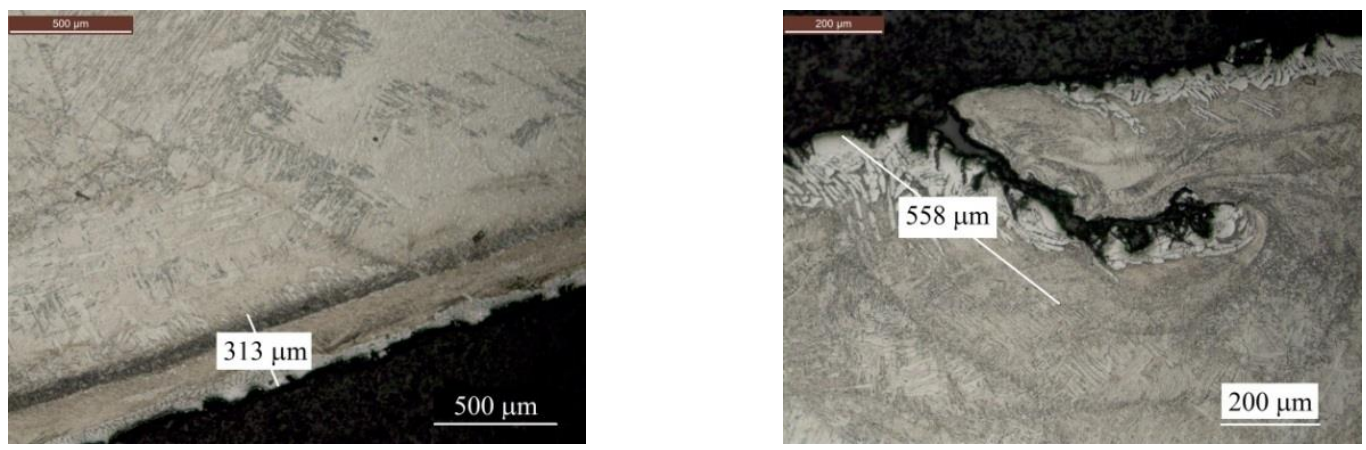

Figura 10: Capa superficial oxidada ( $\alpha$ case). 50X (Izq.) y Fisura de $0,55 \mathrm{~mm}$ de profundidad. 100X (der.) 
En la Figura 11, se muestra la macrografía longitudinal de una colada de extrusión (material que queda sin extrudar) en las que se aprecia cómo es el flujo del material. Se pueden identificar las estructuras características de la deformación: zona muerta (adyacente a la matriz), el material base y la zona de deformación $[14, \underline{15}]$. También se muestra la formación de un defecto llamado "pipe".

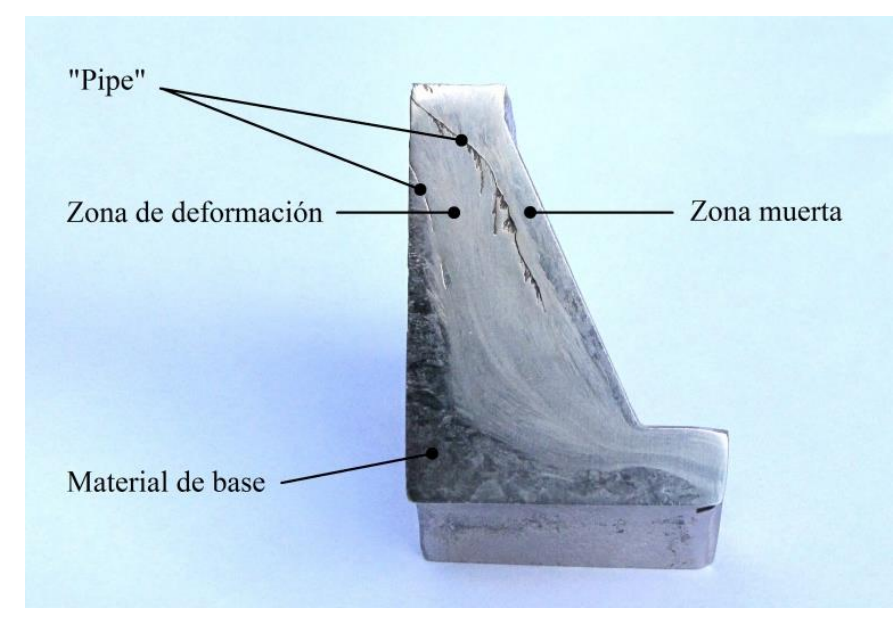

Figura 11: Macrografías longitudinales de coladas de extrusión para perforados de $71 \mathrm{~mm}$

\subsubsection{Aplastado y abocardado}

Se realizaron ensayos de aplastado y abocardado, según la norma ASTM B-338, sobre probetas mecanizadas.

Los resultados indicaron que las probetas de Titanio Gr. 2, en ambos procesos superaron el mínimo del 20\% exigido por norma, sin fisurarse. Mientras que las de Titanio Gr. 5 se fracturaron. Se repitió el ensayo sobre muestras recocidas, las que mostraron una mejora parcial ya que las probetas sólo se fisuraron. El tratamiento térmico de recocido que se realizó fue a la temperatura de $750^{\circ} \mathrm{C}$ con un tiempo de permanencia de 3 horas.

\subsubsection{Tracción}

En todos los casos se realizaron 3 (tres) ensayos por cada muestra. Los resultados obtenidos (promedio) se muestran en la Tabla 3 (laminados perforados) y en la Tabla 4 (extrudados).

Tabla 3: Propiedades mecánicas de los laminados perforados.

\begin{tabular}{|c|c|c|c|c|}
\hline Aleación & Probeta & $\begin{array}{c}\text { Elongación } \\
(\mathrm{lo}=25 \mathrm{~mm}) \\
\%\end{array}$ & $\begin{array}{c}\text { Tensión de Fluencia } \\
\mathrm{MPa}\end{array}$ & $\begin{array}{l}\text { Tensión de rotura } \\
\qquad \mathrm{MPa}\end{array}$ \\
\hline \multirow{3}{*}{ Titanio Gr. 2} & ASTM B-338 & 20 & $275-450$ & $>345$ \\
\hline & 1 & 22 & 347 & 527 \\
\hline & 2 & 21 & 375 & 527 \\
\hline \multirow{4}{*}{ Titanio Gr. 5} & ASTM B-861 & 10 & $>828$ & $>895$ \\
\hline & 3 & 5 & 805 & 1027 \\
\hline & 4 & 7 & 791 & 1069 \\
\hline & 5 (Recocido) & 10 & 756 & 882 \\
\hline
\end{tabular}

Tabla 4: Propiedades mecánicas de los extrudados.

\begin{tabular}{|c|c|c|c|}
\hline Probeta & $\begin{array}{c}\text { Elongación } \\
(\mathrm{lo}=25 \mathrm{~mm}) \\
\%\end{array}$ & $\begin{array}{c}\text { Tensión de Fluencia } \\
\mathrm{MPa}\end{array}$ & $\begin{array}{c}\text { Tensión de rotura } \\
\mathrm{MPa}\end{array}$ \\
\hline ASTM B-338 & 20 & $275-450$ & $>345$ \\
\hline Titanio Gr. 2 (Diám. 91 mm) & 44 & 313 & 399 \\
\hline Titanio Gr. 2 (Diám. $71 \mathrm{~mm}$ ) & 51 & 248 & 344 \\
\hline
\end{tabular}

La dispersión de los valores de los ensayos realizados sobre las probetas de Titanio Gr. 2 estuvo por 
debajo del 5\%. Mientras que en los ensayos practicados sobre muestras de Titanio Gr. 5, sólo los valores de tensiones de fluencia y rotura estuvieron por debajo del $5 \%$ ya que la elongación tuvo una dispersión del orden del $8.5 \%$.

Los ensayos de tracción se realizaron según norma ASTM E8. Las probetas que se obtuvieron directamente de los semielaborados mediante corte por agua, fueron fresadas hasta las dimensiones finales.

\subsubsection{Dureza}

Se realizaron ensayos de dureza en ambos semielaborados y de ambas aleaciones, utilizando la escala HRB para el Titanio Gr. 2 y la escala HRC para el Titanio Gr.5. La medición de la dureza se realizó en 4 (cuatro) puntos $\left(0^{\circ}, 90^{\circ}, 180^{\circ}\right.$ y $\left.270^{\circ}\right)$ de una sección transversal sobre 5 (cinco) muestras de cada material y proceso. En todos los casos la distribución de dureza fue homogénea ya que la dispersión en cada sección y entre las muestras no superó el 3\% para el Titanio Gr. 2 y el 5\% para el Titanio Gr. 5.

Los resultados de las mediciones (promedio) se indican en la Tabla 5.

Tabla 5: Valores de dureza medidos en cortes longitudinales.

\begin{tabular}{lccc}
\hline \multirow{1}{*}{ Sector } & \multicolumn{2}{c}{ Titanio Gr. 2 } & Titanio Gr. 5 \\
& $\begin{array}{c}\text { Extrudado } \\
\text { HRB }\end{array}$ & $\begin{array}{c}\text { Laminado } \\
\text { HRB }\end{array}$ & $\begin{array}{c}\text { Laminado } \\
\text { HRC }\end{array}$ \\
\hline Pared Exterior & 87 & 92 & 37 \\
Centro & 82 & 88 & 35 \\
Pared Interior & 86 & 88 & 33 \\
\hline
\end{tabular}

\section{CONCLUSIONES}

De los ensayos realizados en ambos semielaborados (perforación laminación y extrusión) de aleaciones de titanio para su caracterización, se pueden extraer las siguientes conclusiones:

1- La inspección visual permitió observar y clasificar varios defectos en ambos procesos, cuya influencia en las propiedades finales del producto exigen que sean eliminados o disminuidos, ya sea por mecanizado, decapado químico u otro procedimiento estándar, antes de continuar con su procesamiento.

2- Los defectos encontrados están asociados no sólo al material sino a los parámetros dependientes del proceso. Debe considerarse el diseño del herramental para que el material fluya a través de la zona de deformación minimizando la formación de defectos, tanto externos como internos.

3- Según las normas internacionales, ASTM B338 y B861, para tubos con y sin costura, los semielaborados de Titanio Gr. 2 y Titanio Gr. 5, no se encuadran bajo estas normas. Sin embargo un posterior retrabajado (mecanizado, granallado o decapado químico) sumado a un posterior proceso termomecánico permitirá obtener los requerimientos establecidos en estas normas para ambas aleaciones.

4- La excentricidad se ve afectada por la heterogeneidad de la temperatura del material y por una correcta puesta a punto de los equipos. No es posible reducir la excentricidad en las etapas posteriores de deformación salvo que el semielaborado se mecanice interna y externamente, representando un aumento de los costos por pérdida de material que será considerable cuanta más alta sea la excentricidad.

5- El estudio metalográfico permitió evaluar estructuras y los distintos flujos de material en cada uno de los procesos. Esto es de suma importancia en el diseño del herramental y la reducción de defectos.

6- Los análisis químicos determinaron un aumento de la contaminación gaseosa superficial debido a la atmósfera del horno durante el calentamiento. En el caso del Titanio puro el alargamiento se reduce a la mitad cuando el nivel de oxígeno es del orden del $0,40 \%$ y esto podría producir fisuras superficiales durante la deformación en frío.

7- Los ensayos mecánicos de aplastado y abocardado de los semielaborados de Titanio Gr. 2, de ambos procesos, predicen una alta ductilidad o deformabilidad en frío para el Titanio Gr. 2. Mientras que para el Titanio Gr. 5, dada su baja performance es necesaria la aplicación de un proceso de deformación gradual. 


\section{AGRADECIMIENTOS}

A los Ings. Martín Valdez, Roberto Casanovas y Guillermo Pantiu de Tenaris Siderca S. A.. A los Ings. Germán Tretiak y Roberto Martínez de FV S. A.- Grifería de alta tecnología.

Al Sr. Ricardo Correa de Metalcris S. A..

\section{BIBLIOGRAFÍA}

[1] MERLONE G., NUÑEZ PETTINARI S. I., Atlas de Trabajabilidad en Caliente de Aceros. Sector Laminación, Dirección de Investigaciones Industriales, IAS. Div. Trat. Térmicos, Dto de Materiales, CNEA. 1994.

[2] DIETER G., Mechanical Metallurgy. Mc Graw-Hill, Inc. Tercera Edición, Estados Unidos, 1976.

[3] PRASAD, Y., SASIDHARA, S., Hot Working Guide: A Compendium of Processing Maps, ASM International. Materials Park, Ohio, Estados Unidos, 1997.

[4] REALE, H., COCCO, R., Introducción a las aleaciones de Titanio, Presentación, FAESA.

[5] BOYER, R., WELSCH, G., COLlingS, E., Materials Properties Handbook: Titanium Alloys, ASM International. Materials Park, Ohio, United States of America, 1994.

[6] DONACHIE M., Titanium. A Technical Guide. ASM International, Materials Park, Ohio, Estados Unidos. 1988.

[7] CARR, G., Caracterización Tribológica y Desgaste de Materiales Para la Industria Siderúrgica, División de Soldadura y Fractura de INTEMA, 2008.

[8] CERETTI, E., GIARDINI, C., BRISOTTO, F., "Analytical Prediction of the Central Rupture of Billets During Piercing in a Cross-Roll Piercer due to the Mannesmann effect", In: Presentación, Innovations in Metal Forming - an International Conference, Brescia, Italy, 2004.

[9] DYL, T., Numerical Analysis of the Piercing-spreading Process in Skew Rolling Mill, Institute of Modelling and Automation of Plastic Forming Processes, Faculty of Materials Processing Technology and Applied Physics, Czstochowa Technical University, Al Armii Krajowej 19, 42 - 200 Cz stochowa, Poland.

[10] BAUSER, M., SAUER, G., SIEGERT, K., Extrusion, ASM International, Materials Park, Ohio, Estados Unidos, 2006.

[11] LAUE, K., STENGER, H., Extrusion: Processes, Machinery, Tooling, ASM International, Materials Park, Ohio, Estados Unidos, 1976.

[12] ASTM B338-13, Standard Specification for Seamless and Welded Titanium and Titanium Alloy Tubes for Condensers and Heat Exchangers, ASTM International, United States, 2013.

[13] ASTM B861-10, Standard Specification for Titanium and Titanium Alloy Seamless Pipe, ASTM International, United States, 2010.

[14] ASM Handbook, Metallography and Microstructures, v.8, ASM International, Materials Park, Ohio, Estados Unidos, 2004.

[15] BARTLO, L., Metallography of Titanium and Titanium Base Alloys, Metals Engineering Institute, Home Study and Extension Courses, ASM International, Materials Park, Ohio, Estados Unidos, 2004. 